\title{
Les langues de spécialité et le langage technique : considérations didactiques
}

\section{Ross Charnock}

\section{(2) OpenEdition}

Édition électronique

URL : http://journals.openedition.org/asp/2566

DOI : 10.4000/asp.2566

ISBN : 978-2-8218-0380-0

ISSN : 2108-6354

\section{Éditeur}

Groupe d'étude et de recherche en anglais de spécialité

Édition imprimée

Date de publication : 1 décembre 1999

Pagination : 281-302

ISSN : 1246-8185

Référence électronique

Ross Charnock, "Les langues de spécialité et le langage technique : considérations didactiques », ASp [En ligne], 23-26 | 1999, mis en ligne le 09 novembre 2011, consulté le 19 avril 2019. URL : http:// journals.openedition.org/asp/2566 ; DOI : 10.4000/asp.2566

Ce document a été généré automatiquement le 19 avril 2019

Tous droits réservés 


\title{
Les langues de spécialité et le langage technique : considérations didactiques
}

\author{
Ross Charnock
}

\section{Introduction}

1 Une des questions les plus importantes pour les enseignants de langues aux spécialistes des autres disciplines concerne la part de la discipline principale à prévoir dans le cursus linguistique. On a coutume de dire que le débat reste ouvert, mais en fait les avis sont tellement divergents que la question est rarement débattue. Il s'agit plutôt d'une polémique.

2 Les uns, voulant se démarquer de l'enseignement scolaire, proposent un enseignement fondé principalement sur la terminologie de la langue spécialisée. D'autres, au contraire, constatant le niveau «scolaire » des étudiants inscrits en langue par obligation, trouvent qu'il est impératif de proposer d'abord un cours de rattrapage linguistique ${ }^{1}$.

3 Les deux approches se heurtent à des objections évidentes. Du point de vue didactique, il est clair que pour étudier une nouvelle matière, au niveau universitaire, en langue étrangère de surcroît, il faut déjà être compétent en langue. De ce point de vue, l'enseignant est amené à supposer déjà acquises les compétences qu'il est censé enseigner. À l'inverse, un programme purement linguistique, fondé inévitablement sur la grammaire classique, ne saurait répondre de manière adéquate aux besoins non plus. En effet, si les méthodes scolaires étaient vraiment efficaces, l'enseignant ne serait pas amené à reprendre les mêmes explications de base, avec les mêmes étudiants, désormais universitaires.

4 Les adversaires dans ce débat, du moins en ce qui concerne les représentants des positions extrêmes, reconnaissent volontiers les faiblesses des différentes approches préconisées. Ceux qui préfèrent se concentrer sur la langue spécialisée, en espérant favoriser l'acquisition par la pratique, sont bien obligés de tenir compte des problèmes qu'ils ne manquent pas de rencontrer, et de proposer le cas échéant des rappels de 
grammaire. À l'inverse, ceux qui se sentent obligés de s'occuper d'abord des problèmes élémentaires doivent nécessairement introduire un contenu dans leur cours. En effet, sans ce contenu, les étudiants ne pourront jamais acquérir les compétences communicatives qui constituent l'objectif de l'enseignement. Quel que soit le point de départ, on aboutit ainsi à un compromis. Malheureusement, le compromis ne constitue pas forcément un idéal pédagogique et ne permet pas d'aboutir à un consensus sur le plan de la didactique linguistique.

$5 \quad$ S'agissant spécifiquement d'un cours de langue, il semble a priori préférable d'élaborer un programme non pas en s'alignant automatiquement sur la discipline principale, mais plutôt en appliquant des critères qui relèvent plus précisément de la didactique linguistique. Pourtant, il ne peut être question non plus d'ignorer la langue de spécialité. $\mathrm{Au}$ contraire, il faudrait essayer de profiter des connaissances spécialisées autant que possible, dans la mesure où cela pourrait faciliter l'acquisition de la langue. La question semble à la fois importante en ce qui concerne la didactique, et complexe sur le plan linguistique. Il faut en effet essayer de réconcilier deux impératifs apparemment contradictoires.

6 Afin de proposer une contribution raisonnée à ce débat, on établira dans un premier temps une distinction entre la notion de langue de spécialité, définie en fonction de la discipline concernée, et celle de langage technique, présenté ici comme un mode d'expression, susceptible de définition linguistique indépendamment du sujet traité. Le même modèle théorique sera ensuite généralisé afin d'élaborer une définition de la facilité linguistique, en rapport étroit avec la technicité. Selon cette analyse, et contrairement à ce que l'on pourrait imaginer, le langage technique semble plus facile, de certains points de vue, que la langue de tous les jours. Enfin, dans la dernière partie, on envisagera quelques conclusions didactiques possibles.

\section{Langues de spécialité}

7 On parle de langue de spécialité lorsqu'il s'agit de se servir d'une langue naturelle (la langue de référence) pour rendre compte de connaissances particulières. Dans ces conditions, il ne peut être question de définition linguistique. Comme le signale Lerat (1997: 2): «aucune théorie linguistique, quelle qu'elle soit, n'a jamais isolé le fonctionnement des langues spécialisées de celui des langues naturelles en général ». Les langues de spécialité semblent fonctionner non pas comme des langues autonomes, ayant chacune ses caractéristiques spécifiques, mais comme des fragments ou des sousensembles de la langue naturelle. Il serait donc étonnant d'y trouver une expression, ou une tournure syntaxique, qui n'existerait pas déjà dans la langue de référence. Il n'est pas question ici de contester l'intérêt ou l'utilité des études terminologiques. Pourtant, étant donné l'absence de critères de définition linguistiques de cette notion, il semble légitime d'émettre des réserves concernant son application pédagogique, et d'exprimer des réticences concernant l'utilité de la langue de spécialité pour l'enseignement de la langue.

Il est certes possible non pas de définir, mais plutôt de reconnaître les langues de spécialité, par exemple par la présence d'un vocabulaire particulier, ou par la fréquence d'occurrence d'unités dites terminologiques. Une telle approche a cependant plusieurs inconvénients. Premièrement, il existe des termes spécialisés, en rapport avec des concepts spécifiques, pour chaque domaine d'activité humaine, y compris des activités qui n'ont rien de technique. À l'aide de ces termes, il est possible de rendre compte des 
connaissances des interlocuteurs dans toutes les situations de discours (au bureau, dans les transports en commun, en famille, pour le bricolage, pour le sport, et ainsi de suite), ce qui pourrait justifier l'idée d'autant de langues spécialisées. Tout discours devrait alors être qualifié de spécialisé et la notion deviendrait inopérante.

D'autre part, il n'existe pas de définition linguistique permettant de distinguer les « unités technologiques » des unités lexicales de la langue ordinaire. À la différence des "termes savants", reconnus par leur statut étymologique particulier (cf. Mortureux 1995). Les termes techniques ne sont associés à aucun critère morphologique ou lexicologique spécifique. Il semble au contraire qu'ils aient la même structure morphologique que les unités de la langue de base (Desmet $1998: 296$; voir aussi Humbley 1997).

D'autre part, sur le plan de la sémantique, même lorsque ces termes relèvent clairement d'une discipline donnée, ils ne dénotent pas forcément des concepts précis. Dans les textes récents sur l'économie politique, par exemple, on parle souvent de sustainable growth. Il s'agit d'un terme qui n'est pas susceptible de définition technique, et qui pourrait même être qualifié, par les plus pessimistes, d'oxymore. Il en va de même pour open government, dont il est parfois question en science politique. Bien que spécialisés, ces termes ne peuvent donc pas être considérés comme techniques, pas plus que le terme suit , utilisé de manière métonymique par les informaticiens américains pour signaler la présence des responsables de la comptabilité, ceux-ci étant toujours convenablement habillés.

11 En outre, le statut des concepts « techniques » dénotés par ces termes est loin d'être clair. Pour les uns, un discours donné peut sembler quasi incompréhensible, à cause de la haute technicité de la matière ; cependant, le même texte ne semble poser aucun problème pour d'autres, qui considèrent que les mêmes concepts relèvent plutôt des connaissances générales. D'autre part, de nombreux termes, réservés au départ aux seuls initiés, peuvent passer dans la langue courante au bout d'un laps de temps assez court. Si les découvertes scientifiques trouvent des applications auprès du grand public, les termes associés seront vite adoptés dans le discours de tous les jours. De même, si on peut expliquer des effets économiques réels à l'aide de concepts abstraits, on ne manquera pas d'y faire allusion dans les quotidiens non spécialisés.

12 Enfin, si on reconnaît les langues de spécialité par la présence de termes associés à une discipline particulière, il faudrait alors admettre comme langue spécialisée des discours qui relèvent de styles ou de modes d'expression très variés, allant du registre soutenu à celui de la conversation de tous les jours. Puisque les textes de vulgarisation utilisent les mêmes termes techniques, il faudrait considérer ces textes comme tout aussi spécialisés que les véritables articles de recherche, même si les termes dont il s'agit sont mentionnés ou cités, plutôt qu'employés à des fins véritablement techniques.

Il est à noter ici que, malgré l'absence de définition précise, la plupart des terminologues semblent réserver la notion de langue de spécialité à des secteurs spécifiques, considérés intuitivement comme technologiques ou du moins techniques. De ce point de vue, les langues spécialisées correspondent à la notion de langage technique, telle qu'elle sera présentée ici. En revanche, la plupart des enseignants de langue n'hésitent pas à qualifier d'« anglais économique », par exemple, des textes journalistiques, donc non techniques, tirés des quotidiens ou de revues généralistes. En l'absence de critères linguistiques de définition, il est difficile d'éviter ce type de désaccord. Il est donc nécessaire de distinguer 
entre les notions de langue de spécialité et de langage technique, en proposant une définition linguistique de la technicité.

\section{La technicité}

14 Intuitivement, le langage technique semble facile à reconnaître. On s'accorde généralement pour dire que les auteurs des articles techniques et scientifiques s'expriment d'une manière aussi explicite et précise que possible. On remarque habituellement l'exactitude et la clarté de ce mode d'expression. Pourtant, malgré cette impression de clarté, on constate aussi un certain manque de lisibilité, ou de confort de lecture. Une présentation théorique de cette notion doit rendre compte de manière cohérente de toutes ces intuitions, en définissant le langage technique comme un mode d'expression linguistique, indépendamment de la discipline ou du domaine.

15 L'analyse proposée ici dépend de la distinction fondamentale opérée en linguistique pragmatique entre la phrase et l'énoncé. L'énoncé, le fait observable, est associé par définition à un contexte d'énonciation, alors que la phrase sous-jacente existe à un niveau plus abstrait, hors contexte. Il est à noter que ces notions ne correspondent pas aux concepts grammaticaux classiques; il s'agit plutôt de niveaux de connaissance ou de compréhension linguistique.

16 Selon les conventions terminologiques ${ }^{2}$, la phrase est associée à une signification (sémantique), alors que l'énoncé véhicule un sens intentionnel, en rapport avec le contexte. Il n'est pas question d'une opposition binaire entre les deux niveaux, puisqu'ils sont tous les deux nécessaires pour la communication. D'une part, on ne peut communiquer uniquement au niveau du sens intentionnel, sans rapport perceptible entre les mots et le message. Et de l'autre, il faut admettre en même temps l'impossibilité de communication au niveau purement littéral, sans prise en compte du contexte (cf. Searle 1979). Dans la théorie de la pragmatique intégrée, le sens et la signification sont considérés, pour des raisons épistémologiques, comme interdépendants ${ }^{3}$.

Puisque, par définition, il ne peut y avoir d'énonciation sans contexte, la connaissance de la signification ne peut jamais suffire pour la compréhension de l'énoncé. En d'autres termes, le sens et la signification ne peuvent jamais être identiques. Néanmoins, pour les besoins de l'exposé, on dira que le rôle du contexte peut être plus ou moins important, selon les cas. En effet, certains textes (techniques) énoncent des vérités génériques, de manière impersonnelle, dans un but communicatif clair et précis, et de façon aussi explicite que possible; le rôle du contexte est alors réduit au minimum. D'autres textes (non techniques) s'expriment à un niveau plus personnel, par sous-entendus, ou en évoquant des références culturelles. La compréhension exige alors une connaissance détaillée du contexte et de la situation extralinguistique. Puisque toutes les positions intermédiaires sont également possibles, on est amené à poser l'existence d'un continuum. Pour rendre compte de l'importance variable du contexte, on dira que le sens peut être plus ou moins éloigné de la signification. Selon la définition proposée ici, lorsque le mode d'expression est technique, la divergence entre le sens et la signification est réduite au minimum. Étant donné que cette divergence est variable, il faut alors admettre l'existence de degrés de technicité.

L'analyse stylistique et discursive permettra de montrer, d'une part, que cette définition du langage technique correspond bien aux notions intuitives correspondantes à ce mode 
d'expression et, de l'autre, qu'il s'agit effectivement d'une définition linguistique, qui s'applique indépendamment du contenu. Il apparaîtra alors que si le langage technique est parfaitement adapté aux sujets scientifiques et technologiques, son emploi n'est pas limité pour autant à ces disciplines. En effet, tout sujet, même apparemment banal, peut être traité de manière technique; à l'inverse, il est tout à fait possible d'employer d'autres styles même lorsqu'il s'agit d'évoquer des sujets spécialisés.

\section{Cadre d'analyse}

Il est commode de faire apparaître l'importance variable du contexte par une analyse en fonction de trois paramètres : la cohésion, la cohérence et la connotation. Selon Halliday \& Hasan, la cohésion fonctionne au niveau de la phrase, lorsque « the interpretation of some element in the discourse is dependent on that of another ». (1976:4)

Cela concerne par exemple l'emploi des pronoms ou des déterminants, ou alors la répétition, la substitution et les ellipses. Puisqu'il s'agit en principe d'interpréter le texte, au niveau de la phrase, la cohésion doit être considérée comme un phénomène sémantique, en rapport avec la signification. En revanche, le terme de cohérence est employé par Widdowson (1979: 87) pour désigner: «the link between the communicative acts that sentences are used to perform ». Cela concerne d'une part les sous-entendus et de l'autre la continuité des enchaînements thème/rhème (ou «topic/comment»). Il s'agit cette fois d'un phénomène pragmatique et contextuel, qui fonctionne au niveau de l'énoncé. Enfin, on utilisera le terme de connotation lorsque le message porte surtout sur l'aspect non dénotationnel des termes. On regroupera dans cette catégorie les emplois figuratifs ou métaphoriques, les cas où le sens reste indéterminé, et les références culturelles.

21 Ces trois paramètres ne sont pas totalement indépendants les uns des autres. L'ellipse, par exemple, est parfois syntaxiquement obligatoire et doit alors être traitée au niveau de la cohésion. Cependant, son interprétation peut également nécessiter des inférences pragmatiques, dont il faut tenir compte au niveau de la cohérence. De même, la substitution coréférentielle, phénomène grammatical, est souvent utilisée pour traduire des valeurs et des jugements personnels, traités au niveau de la connotation. On remarquera que, de manière générale, plus la cohésion est explicite, moins il y aura besoin d'inférences pragmatiques au niveau de la cohérence.

Dans le cadre d'un article assez court, il ne peut être question d'une analyse détaillée, à partir d'un corpus complet. Les quelques extraits figurant en annexe permettront cependant d'illustrer certains des points les plus évidents. Il sera possible, à l'aide de ces différents paramètres, de faire apparaître la divergence variable entre le sens et la signification selon le mode d'expression linguistique, et ainsi de souligner l'importance des critères pragmatiques, au niveau théorique et au niveau de l'interprétation.

\section{Cohésion}

Selon l'hypothèse à vérifier ici, les relations discursives sont présentées, dans le langage technique, de manière aussi claire et explicite que possible, la divergence entre le sens et la signification étant ainsi réduite au minimum. Étant donné que l'interprétation des pronoms et des relations anaphoriques dépend par définition de la compréhension 
d'autres termes figurant dans le discours, il n'est pas surprenant de constater que, dans le langage technique, leur emploi est réduit au minimum. La répétition y est systématiquement préférée. Dans la première phrase du texte 4 , par exemple, l'expression intellectual property rights est répétée, ce qu'on aurait pu éviter en utilisant they ou the former. De même, dans le texte 3, en français juridique, l'auteur anonyme préfère répéter l'expression complexe les femmes de débauche ou les individus de mœurs spéciales, plutôt que d'employer les pronoms ils ou elles. Pour éviter tout risque de confusion, le rapport anaphorique est rendu encore plus encore explicite à l'aide de l'expression visés au paragraphe précédent, sans que cette précision ne soit nullement nécessaire. On peut également remarquer que dans le texte 7, Keynes préfère répéter cinq fois l'adjectif aggregate, afin de faciliter la compréhension de son texte, plutôt que de laisser la portée de l'adjectif à l'appréciation du lecteur. Il ne s'agit pas ici d'un élément stylistique, mais d'un critère constitutif du langage technique.

On ne nie pas la présence de pronoms dans les textes techniques, même si on peut constater que ceux-ci ne sont utilisés que lorsque la référence est clairement fixée. Dans des textes 2, 7 et 11, par exemple, les auteurs sont désignés de manière déictique par les pronoms I, ou we. Les relations cohésives semblent tout aussi claires dans le texte 5 , où le she, de She had lodged..., désigne S. Liebeck, et dans le texte 6, où le him, de brave of him, renvoie à $C$. Higson. Cependant, dans le style journalistique, la fixation de la référence n'est pas toujours aussi simple. Il est souvent nécessaire de reconstruire des concepts complexes qui ne figurent pas explicitement dans le discours-avant. Ainsi, dans reduce them et it can be changed (texte 9), le them renvoie à interest rates, alors que le it renvoie à monetary policy. Dans le dialogue (texte 12), les pronoms déictiques font référence à la situation extralinguistique, souvent de manière peu claire.

La substitution est quelquefois grammaticalement nécessaire; ce phénomène n'est donc pas exclu du langage technique. Dans le texte 2, le do, de to do so, remplace extend to smaller lengths, alors que le one, de a bad one, dans le texte 9 , renvoie à argument. En revanche, ce n'est que dans le langage non technique qu'on pourra trouver des exemples de substitution coréférentielle pour des raisons purement stylistiques. Dans le texte 5 , l'expression the company est utilisée à la place de McDonald's, apparemment pour assurer une certaine variété discursive.

On constate sans surprise que lorsqu'elles ne sont pas grammaticalement obligatoires, les ellipses sont évitées dans le langage technique. Elles sont pourtant courantes dans le langage non technique, par exemple dans le texte 10, lorsqu'il faut comprendre, comme sujet du verbe tend dans and so tend to ignore, l'expression most contemporary economic theories. Dans le même texte, il faut comprendre, après believed dans one of the most widely believed is that... l'expression criticisms made of economics.

La préférence pour la répétition et le refus de l'ellipse ont des conséquences intéressantes pour l'emploi du mot this dans le discours technique. En effet, dans le langage technique, this a quasi exclusivement la fonction, non pas de pronom démonstratif, mais de déterminant, ce qui implique la répétition du substantif associé. On trouve dans le texte 2 (anglais scientifique) this continuity, où le mot continuity figure déjà dans le discoursavant. Dans le texte 11 on trouve this article, mais il s'agit là d'une référence déictique, autoréférentielle. Dans les textes scientifiques et techniques, on ne trouve des exemples de this, utilisé comme pronom, en remplacement d'un nom, que lorsque son emploi est grammaticalement obligatoire. C'est le cas de extend this, dans le texte 2, où le this fait référence à une distance minimale qui vient d'être mentionnée. De la même façon, dans le 
texte 7, de Keynes, le this de I mean by this..., est en rapport direct avec general theory. En revanche, dans le langage non technique, this est employé beaucoup plus facilement comme pronom démonstratif, sans lien direct avec un référent précis dans le discoursavant. Ainsi, dans le texte 9 , dans This is what Keynes called..., le this fait référence à une situation économique assez complexe, longuement décrite dans le paragraphe précédent. De même, dans le texte 6 , le this de This was brave of him... fait référence à un acte difficilement concevable, dont on préfère ne pas connaître les détails. Il est vrai qu'on trouve également un this, pronom démonstratif, dans le texte 11, de Russell : (This is not so strictly what is meant as...), mais il s'agit d'un commentaire, placé entre parenthèses par l'auteur lui-même, qui ne fait pas partie de la démonstration technique proprement dite.

\section{Cohérence}

Afin de minimiser l'importance des inférences pragmatiques nécessaires pour l'interprétation, les enchaînements thème/rhème sont généralement présentés, dans le langage technique, de manière claire et explicite. Même lorsque l'expression désignée comme thème ne figure pas textuellement dans le contexte linguistique, elle est toujours facilement récupérable. Dans le texte 2, par exemple, le rapport semble clair entre Les peines ci-dessus, au deuxième paragraphe, et l'emprisonnement ... et ... l'amende dans le paragraphe précédent ; en effet, ces termes sont en rapport hyponymique. En revanche, dans le langage non technique, des inférences pragmatiques d'une plus grande complexité sont souvent nécessaires pour saisir la continuité du texte. Dans le texte 6 , à partir de little noticed judgement, on enchaine sur the case; puis, à partir de gaoled for two years on enchaîne sur the sentence. De même, dans le texte 5 , pour suivre la continuité du discours, il faut saisir le rapport entre lodged the cup between her thighs et spilt a cup of coffee , rapport qui n'est explicité nulle part dans le texte. Il ne s'agit pas simplement du remplacement d'un terme par un autre, mais de la mise en œuvre d'un scénario dont la compréhension dépend des connaissances extralinguistiques. Toujours dans le texte 5 , le rapport entre tort cases, dans le deuxième paragraphe, et l'affaire décrite quelques lignes plus haut n'est pas explicité non plus. Enfin, la cohérence semble particulièrement difficile à appréhender dans le texte 10 ; on trouve dans le deuxième paragraphe une suite de sept syntagmes nominaux, apparemment sans rapport les uns aux autres. Pour saisir le point commun, il faut connaître la fin du paragraphe ; après relecture, on comprend que, selon l'auteur, il s'agit de phénomènes qui relèvent tous du irrational behaviour.

De même, dans le discours technique, la structure rhétorique est souvent marquée de manière explicite. Certes, l'explicitation des actes rhétoriques amène inévitablement une certaine complexité au niveau de la phrase ; mais loin de rendre la tache du lecteur plus difficile, l'objectif est justement de faciliter l'interprétation en guidant les attentes. Dans le texte 7, les expressions I mean by this that..., et I argue that, permettent de faciliter la compréhension en précisant l'orientation argumentative. Dans le texte 8, le discours est structuré de manière explicite par We turn now to ..., puis par I posit ..., le but étant précisé à l'avance (To locate the derivation ...). Dans le texte 11, l'auteur indique son point de vue de manière tout aussi claire (the view which I advocate). Il précise en outre que la dernière phrase, entre guillemets, est l'abréviation d'une affirmation donnée en début d'article, ce qui ne peut que faciliter la compréhension.

On voit ainsi que la complexité syntaxique associée au langage technique ne semble pas poser de problèmes d'interprétation particuliers. Au contraire, la phrase complexe se 
présente à la lecture comme une suite de phrases simples, articulées de manière cohérente. De plus, il est rare de trouver, dans le langage technique, des informations nouvelles dans les subordonnées. Elles servent surtout pour rappeler des faits déjà mentionnés, ou des informations faisant partie des connaissances communes. Ainsi, dans le texte 8 , l'expression dépendante With the derivative security characteristics fully specified ne fait que rappeler ce qui vient d'être dit. Dans le texte 2, les scientifiques compétents seront tous d'accord à l'avance avec le contenu de la phrase relative which is a condition so extreme....

31 En revanche, dans le langage non technique, on trouve souvent des informations nouvelles présentées sous forme de présupposés. En lisant his wife, dans le texte 5 , le lecteur apprend (non sans un certain étonnement) que l'accusé est marié. De la même façon, des informations nouvelles sont souvent véhiculées par sous-entendus, comme dans le texte 12, où la présence de two mugs on that shelf laisse entendre la présence d'une seconde personne dans la maison. Si l'interlocuteur lui-même ne saisit pas tout de suite ce raisonnement (il semble plutôt interpréter l'énoncé comme une requête), cela ne fait que manifester la complexité du processus inférentiel nécessaire pour l'interprétation. Le People. Person, à la réplique suivante, qui relève de l'auto-correction, souligne la confusion de l'interlocuteur. De même, dans le langage non technique, on trouve souvent de nouvelles informations dans les phrases subordonnées, comme c'est le cas avec le unimpressed (texte 6), qui semble être un bel exemple de litote. Dans le langage technique, on évite de véhiculer des informations nouvelles de cette manière ${ }^{4}$.

\section{Connotation}

32 En principe, les termes techniques correspondent à des concepts précis. Dans la mesure où ils désignent des concepts spécifiques, ils peuvent être employés de manière purement référentielle. Pour les spécialistes, ces termes ne posent donc pas de problèmes particuliers d'interprétation.

En revanche, on trouve souvent dans les textes journalistiques des expressions dont la définition est laissée à l'imagination du lecteur, qui doit par exemple reconstruire le sens de l'expression homo economicus (texte 10), à partir du contexte linguistique (ici l'opposition avec « homo sapiens »).

Les expressions non définies sont couramment utilisées, dans le langage non technique, pour évoquer des idées et des impressions subjectives. C'est le cas de brave, ou de disgust dans le texte 6 , expressions qui font apparaître les réactions personnelles du juge ; il en va de même pour virtues, ou pour "doves " (mot placé entre guillemets par l'auteur) dans le texte 9. Le sens de dangerous, dans le texte 12, reste peu clair, même dans le contexte donné. De telles expressions sont souvent introduites par substitution, pour désigner sous un nouvel aspect un concept déjà présent dans le discours. Ainsi, dans le texte 9 , l'auteur revient sciemment sur la notion de business cycles en utilisant d'abord l'expression temporary recession, puis long-lasting slump.

On a déjà vu que sous certaines conditions, les termes techniques peuvent passer de la langue de spécialité à la langue de tous les jours. Ils sont alors employés de manière moins précise. En revanche, d'autres expressions, utilisées couramment dans le langage ordinaire, peuvent être adoptées par les spécialistes comme termes techniques, en relation univoque avec un concept précis. Les mêmes termes sont alors entendus 
différemment par le spécialiste et le non-spécialiste. C'est le cas de délinquant, des femmes de débauche, ou des individus de mours spéciales dans le texte 3 ; il en va de même pour abuse dans le texte 4 et pour punitive award dans le texte 5 .

D'autres termes semblent non pas ambigus, mais vagues et indéterminés ; ils sont utilisés pour désigner des concepts fondamentalement imprécis, par exemple lorsqu'il s'agit de décrire un phénomène scientifique dont on ne maitrise pas tous les détails. Dans le texte 11, on trouve par exemple essentially, qui souligne un certain manque de précision. Dans le texte 2, on trouve aussi about (qui désigne une distance très courte), of that order, et so extreme. Notons que les auteurs emploient une deuxième fois about dans le même paragraphe lors de la définition d'une condition qui est completely beyond our present knowledge. Ils ont aussi plusieurs fois recours à la modalité (it may be), pour exprimer une possibilité plutôt qu'une certitude.

On trouve également dans le langage non technique des expressions idiomatiques ou figuratives, des références culturelles externes et des interventions personnelles. Par définition, le sens des expressions idiomatiques n'est pas prévisible à partir de la signification des mots qui les composent. Ces expressions, généralement évitées dans le langage technique, sont courantes dans la langue générale. Dans le texte 10, on trouve par exemple what makes people tick, et part and parcel. Dans le texte 9, on trouve pushing at a piece of string (expression placée entre guillemets, et censée être co-référentielle avec «liquidity trap »), ainsi qu'une variante à partir de l'expression figée « egg on face » (political egg on anyone's face).

D'autres expressions évoquent, sans nécessairement les citer directement, d'autres textes et d'autres discours, supposés connus du lecteur. Le sens de ces expressions est très difficile à cerner, et leur interprétation requiert souvent une certaine culture littéraire. On en trouve non seulement dans le discours érudit, mais aussi dans des commentaires journalistiques, et dans la conversation courante. Dans le texte 10, l'expression dismal science, co-référentielle avec "economics", renvoie à la célèbre définition proposée à l'origine par Carlyle. De même, l'adverbe brutishly évoque une citation de Hobbes ( «nasty, brutish and short»). De telles expressions servent essentiellement pour transmettre des attitudes subjectives ; il serait donc étonnant d'en trouver dans des textes techniques.

Puisque le langage technique se fonde, dans la mesure du possible, sur la signification, au niveau de la phrase, il est rare d'y trouver les interventions purement personnelles, qui relèvent par définition du sens intentionnel. Les interventions subjectives sont pourtant courantes dans d'autres types de textes, ou dans la conversation courante. Dans le texte 1, par exemple, le Ah, dernier détail, laisse entendre que l'auteur a failli oublier quelque chose.

40 Bien sûr, une analyse plus poussée, par exemple à propos de l'emploi des connecteurs argumentatifs, des verbes modaux, ou des contraintes rhétoriques, pourrait faire apparaître d'autres caractéristiques propres au langage technique. Une recherche plus approfondie pourrait aussi démontrer l'existence de caractéristiques linguistiques spécifiques aux différentes disciplines, caractéristiques dont on reconnaît volontiers l'intérêt. Néanmoins, l'existence de tels points spécifiques n'oblige pas à rejeter la notion plus générale de technicité linguistique.

41 Le modèle pragmatique, utilisé ici pour les besoins d'une analyse essentiellement stylistique, a permis de montrer que le langage technique, contrairement à la langue de spécialité, est bien susceptible de définition linguistique, indépendamment de la langue 
de référence et de la discipline concernée. On constate ainsi l'absence de corrélation entre les notions de langage technique et de langue de spécialité. Le texte 2, par exemple, qui porte sur la physique moderne, correspond à la fois au langage technique et à la langue de spécialité. En revanche, le texte 1 n'a rien de technique, malgré la présence de nombreux termes qui relèvent de l'informatique ${ }^{5}$. On voit ainsi que le mode d'expression de la langue de spécialité peut-être, mais n'est pas nécessairement, technique.

L'idée de divergence variable entre le sens et la signification permet également de rendre compte de l'existence des différents degrés de technicité, et de considérer les articles de recherche comme plus techniques que les textes de vulgarisation, même lorsque ceux-ci relèvent de la même spécialité. Parmi les quatre textes comportant des termes juridiques (3 à 6), deux seulement (3 et 4) correspondent à la définition du langage technique, les deux autres (5 et 6 ) pouvant plutôt être qualifiés de journalistiques. De même, deux seulement des quatre textes sur des sujets économiques peuvent être considérés comme techniques. Il s'agit des textes 7 et 8 ; les deux autres ( 9 et 10) sont à l'évidence des textes journalistiques, le 10 se plaçant particulièrement bas dans l'échelle de la technicité.

Remarquons enfin qu'il est tout à fait possible de trouver les caractéristiques du langage technique même en l'absence de termes techniques. On peut trouver par exemple chez les philosophes du langage des analyses savantes et détaillées de concepts qui relèvent de la vie quotidienne. C'est le cas du texte 11.

\section{La facilité du langage technique}

La présentation généralisée du langage technique semble être corroborée par les recherches empiriques menées par plusieurs auteurs, qui ont constaté de manière indépendante que les besoins linguistiques des chercheurs ne varient pas selon la discipline.

Cooke (1993: 470) signale l'existence d'un «common core of unknown or poorly acquired elements ", alors que Birch fait remarquer, dans son étude sur les problèmes de rédaction rencontrés par le chercheurs «non-natifs », que :

[...] the same sort of modifications are required from author to author, and it seems that this may hold for researchers working in different domains and with varying levels of language skills. $(1996: 76)^{6}$

Il convient de noter que les problèmes linguistiques dont il est question dans les deux articles relèvent toujours de la langue ordinaire plutôt que de la langue de spécialité.

Cette analyse est confirmée par Hutchinson \& Waters (1987: 161), qui en tirent une conclusion pédagogique importante ; ils considèrent en effet qu'il n'y a pas de différence essentielle entre l'enseignement de la langue pour objectifs spécifiques et l'enseignement de la langue en général. Pour ces auteurs, l'utilisation de textes spécialisés ne serait justifiée qu'au niveau de la validité apparente (face validity).

On parle au pluriel de "langues de spécialité », sans qu'il s'agisse en réalité de langues différentes. En effet, celui qui comprend la langue de tous les jours n'aura pas de problèmes linguistiques particuliers lorsqu'il s'agit d'aborder un sujet technique, à condition toutefois de connaître la discipline. En revanche, celui qui sait lire des articles techniques ne sera pas nécessairement compétent pour maîtriser les expressions familières de la langue de tous les jours. La définition proposée de la technicité permet d'expliquer l'apparente facilité du langage technique par rapport à la langue quotidienne. 

que si la théorie permet des généralisations intéressantes. Le modèle proposé pour l'analyse de la technicité répond bien à cette exigence, puisqu'il permet effectivement de proposer une définition de la facilité linguistique («fluency»), en rapport étroit avec la définition du langage technique. La facilité est habituellement présentée comme un phénomène psychologique; il s'agirait d'une qualité attribuée à ceux qui font preuve de confiance et d'aisance dans la prise de parole, qui se font comprendre sans trop de difficultés, qui parlent sans trop d'hésitations et ainsi de suite. Du point de vue de la didactique linguistique, cette description intuitive présente cependant plusieurs inconvénients. D'une part, cette qualité ne pourrait être attribuée indifféremment à tous les sujets parlants de langue maternelle («native speakers»), car certains font preuve de plus d'aisance que d'autres; et, de l'autre, la facilité serait variable chez un même individu selon les circonstances (la fatigue ou la timidité par exemple pourraient avoir un effet sur sa maîtrise de la langue). L'idée de divergence variable entre le sens et la signification permet d'éviter ces inconvénients en proposant une définition non pas psychologique, mais plutôt linguistique, de la facilité.

Selon l'approche adoptée ici, déjà esquissée dans Charnock (1982), la facilité linguistique correspond à la capacité de mise en relation du sens et de la signification. Elle apparaitt comme une compétence commune aux "natifs », mais qui manque chez les apprenants, qui ont souvent des difficultés pour tenir compte en même temps des deux niveaux. Il est à noter que, selon cette définition, la notion de facilité ne concerne plus uniquement les capacités en expression orale; elle peut être généralisée pour tenir compte aussi des compétences à l'écrit et en compréhension. Ainsi définie, selon des critères linguistiques, la notion de facilité semble susceptible d'applications pédagogiques.

51 Cette approche permet de mieux comprendre les difficultés des étudiants des niveaux intermédiaires lorsqu'ils persistent à faire des fautes « rédhibitoires » sur des points de grammaire qu'ils connaissent pourtant très bien. Ces étudiants peuvent souvent obtenir sans difficulté des notes élevées lors d'un test de grammaire, lorsqu'il s'agit d'appliquer des règles au niveau de la phrase; pourtant, dès qu'ils fixent leur attention sur le sens contextuel, ils ne sont plus en mesure de s'occuper des questions de forme.

permet aussi de mieux expliquer la difficulte du discours argumentatif, par rapport au discours purement factuel. On peut effectivement prévoir que, plus le sens intentionnel est éloigné de la signification sémantique, plus la mise en relation des deux niveaux sera difficile. Puisque l'orientation argumentative relève par définition du sens intentionnel, il n'est pas étonnant de constater la difficulté supplémentaire de ce type de discours. Cette analyse semble être corroborée par la plupart des référentiels (ou descripteurs de niveaux), élaborés à des fins d'évaluation linguistique. Ceux-ci placent systématiquement la "maîtrise du discours argumentatif», la "compréhension de l'ironie » et «l'exploitation des références culturelles » à un niveau plus avancé que la communication purement factuelle. Dans les référentiels du DCL, par exemple, le candidat qui sait «argumenter en utilisant les procédures de structuration propres à ce type de discours » est placé en zone 4 (le niveau le plus élevé), alors que celui qui sait « organiser les éléments essentiellement factuels» se retrouve en zone $2^{7}$. Pourtant, la difficulté supplémentaire ne se trouve ni au niveau de la grammaire, ni à celui du lexique.

Selon cette analyse, les problèmes liés au développement de la facilité linguistique ne se posent donc ni au niveau de la structure, ni à celui des intentions communicatives, mais plutôt dans le rapport qu'il faut établir entre les deux. Cette constatation permet 
d'expliquer, dans une certaine mesure, l'insuffisance, souvent dénoncée, d'une part des méthodes fondées uniquement sur la grammaire, et de l'autre des méthodes dites directes. Howatt (1984) montre en effet que sur une période comprenant plusieurs siècles, ni les méthodes scolaires, ni les méthodes communicatives n'ont permis d'obtenir les résultats escomptés. Cette longue expérience ne peut que confirmer l'importance du rapport entre le sens et la signification pour l'acquisition des compétences linguistiques. Des exercices portant précisément sur la mise en relation de ces deux niveaux sont présentés dans Charnock $(1997,1998)$.

Si on accepte cette analyse, et si on admet également la définition de la technicité proposée plus haut, on est amené à considérer le langage technique comme plus simple, en ce qui concerne les compétences communicatives, que la langue de tous les jours. En effet, dans le langage technique la divergence entre le sens et la signification est minimisée ; la mise en relation des deux niveaux devrait donc être plus facile. On peut néanmoins envisager plusieurs objections à cette conclusion, a priori surprenante. Premièrement, on pourrait soutenir que la vulgarisation constitue par définition une simplification du discours spécialisé. En second lieu, on pourrait faire remarquer la complexité syntaxique incontestable du langage technique. Enfin, troisième objection, on pourrait tout simplement rejeter le modèle théorique en niant l'importance des inférences pragmatiques, présentées comme nécessaires pour la compréhension.

En réponse à la première objection, il convient d'observer que si les articles techniques paraissent difficiles, ce n'est pas à cause d'une apparente opacité linguistique, mais à cause de la complexité inhérente à la discipline. Les textes de ce genre restent difficiles pour les non-initiés, même s'ils connaissent bien la langue. Il ne s'agit donc pas d'un problème linguistique, à proprement parler. Si les textes de vulgarisation semblent plus "faciles" que les articles de recherche, ce n'est pas à cause de la simplification stylistique, mais de la reformulation du contenu. Il ne faut pas confondre la commodité, ou le confort de lecture, avec la compréhension proprement dite. En effet, pour celui qui connaît le sujet, la version « simplifiée » semble moins explicite et soulève de nouveaux problèmes.

Pour la deuxième objection, il faut rappeler que, dans la pratique, la complexité syntaxique ne semble pas poser autant de difficultés de compréhension que les problèmes du temps et de l'aspect, habituellement abordés dès le niveau élémentaire. Il est vrai qu'on ne demande pas aux étudiants de niveau élémentaire de produire des phrases aussi longues, que ce soit à l'écrit ou à l'oral; mais il est normal de constater la plus grande difficulté de la production langagière par rapport à la compréhension.

La troisième objection est plus difficile, car il faut reconnaître que les "natifs ", ou les francophones de niveau équivalent, sont rarement conscients de la complexité des inférences pragmatiques théoriquement nécessaires pour la compréhension. En effet, l'analyse linguistique ne correspond pas forcément à une description des opérations mentales des sujets parlants. Pour défendre la théorie proposée, il convient néanmoins de faire remarquer les difficultés des apprenants de niveau intermédiaire, qui ont souvent l'impression de connaître tous les mots d'un texte sans toutefois comprendre le sens. Même les étudiants plus avancés peuvent avoir des difficultés pour saisir le point de vue de l'auteur lorsqu'il s'exprime par sous-entendus ou avec ironie. Enfin, pour se convaincre de la complexité de la langue de tous les jours, par rapport au langage technique, il suffit de rappeler les difficultés d'interprétation du texte 12. En revanche, dans le langage technique, la clarté de la structure, et les conventions rédactionnelles, 
internationalement valables et strictement appliquées, peuvent faciliter la compréhension de la structure rhétorique et du contenu de l'article.

La terminologie spécialisée peut certes sembler difficile pour les non-initiés, mais il convient de remarquer encore une fois que les spécialistes n'éprouvent que rarement des difficultés avec les termes techniques. En effet, dans la mesure où les concepts sont internationaux, le vocabulaire reste sensiblement le même d'une langue à l'autre. D'autre part, les étudiants spécialistes des autres disciplines auront d'autant moins de problèmes de vocabulaire si la fréquence d'occurrence de ces termes est élevée ; ils ont normalement plus de difficultés avec des mots rares, qui relèvent d'autres sujets ou d'autres registres.

Notons enfin que l'apparente facilité du langage technique semble être confirmée. Lerat, qui signale que :

Paradoxalement, les langues spécialisées (sic) sont relativement simples, parce que

les contraintes lexicales, sémantiques et pragmatiques y sont fortes. (1997:6)

Dans le même ordre d'idées, E. Guyon semble confirmer la réalité de ces contraintes lorsqu'il confie (Guyon \& Guyon) que :

Dès lors que je sors de mon champ de compétence et de curiosité scientifique, j'ai beaucoup plus de mal à m'exprimer en anglais. (1996:3)

61 Si on admet le rapport entre les notions de technicité et de facilité linguistique, on est amené à tirer certaines conclusions concernant la didactique linguistique.

\section{Conclusions didactiques}

62 Le modèle linguistique proposé suggère un certain nombre de conclusions concernant d'une part l'enseignement de la langue ordinaire, et de l'autre, la part de la discipline à prévoir dans le cadre de l'enseignement de la langue aux spécialistes des autres disciplines. En ce qui concerne la langue ordinaire, l'objectif du cours étant l'amélioration des compétences communicatives, il semble nécessaire d'introduire un contenu. En effet, pour développer la capacité de mise en relation de la signification et du sens, il faut pouvoir tenir compte du contexte et des intentions communicatives, véhiculées par le discours en continu. Il semble donc parfaitement inutile de travailler sur des phrases individuelles, hors contexte (ou, a fortiori, sur les listes de mots, terminologiques ou non).

Les conclusions concernant la part de la discipline principale semblent tout aussi claires, dès lors qu'on accepte la nécessité d'un contenu quelconque en cours de langue. En effet, si l'on considère que les spécialistes de la discipline comprennent plus facilement le langage technique que la vulgarisation ou la langue de tous les jours, il faudrait envisager l'utilisation de ce type de texte en début de cursus, avec les étudiants les plus faibles, pour n'aborder que plus tard d'autres textes linguistiquement plus complexes. Il semble ainsi possible d'éviter les problèmes non pertinents, pour se concentrer sur le développement des compétences communicatives.

Bien qu'il s'agisse tout simplement de l'application d'une règle pédagogique fondamentale, selon laquelle il faut toujours commencer par ce qui est simple, cette conclusion va bien évidemment à l'encontre des idées reçues concernant la didactique linguistique. Avant de traiter les objections possibles, il convient de mentionner les avantages présentés par une telle approche.

65 Tout d'abord, l'exploitation du langage technique permet d'éviter l'un des problèmes pédagogiques principaux rencontrés dans l'enseignement des langues aux adultes. En 
effet, les faux-débutants sont souvent découragés par le décalage évident entre leur niveau intellectuel et leurs capacités d'expression en langue étrangère. Le contenu des dialogues authentiques dans la langue quotidienne est souvent banal, et sans véritable intérêt. Lorsqu'on demande aux étudiants de travailler sur des versions simplifiées de ces dialogues, ils peuvent avoir l'impression de perdre leur temps avec des exercices enfantins. Le langage technique, en revanche, permet d'exprimer des vérités profondes d'une manière claire et simple. Les spécialistes de la discipline connaissent mieux que les "natifs" non-spécialistes le vocabulaire approprié et les conventions rhétoriques couramment appliquées dans les textes techniques; cela ne peut que favoriser la motivation et donc l'apprentissage à partir du niveau élémentaire.

En revanche, le langage journalistique comporte des difficultés supplémentaires, souvent sans rapport avec le contenu, et pose des problèmes de compréhension inutiles. À cause de leur structure imprévisible, et les nombreuses expressions idiomatiques utilisées, les textes de ce genre ne sont pas acceptables non plus comme modèle approprié pour l'étude de l'expression écrite. En effet, lorsque les spécialistes des autres disciplines s'expriment en langue étrangère, ils doivent le faire dans un tout autre registre, celui justement des articles techniques.

67 On voit ainsi que la notion linguistique de technicité justifie, sur le plan théorique, et sous certaines conditions, l'utilisation de textes techniques en cours de langue. Le concept de technicité permet de sélectionner le matériel pédagogique selon le niveau de difficulté, en fonction de critères linguistiques, ce qui permet de «tenir compte des thèmes propres aux disciplines enseignées ", sans qu'il soit pour autant nécessaire de suivre aveuglément le programme de la discipline principale. Il faut maintenant traiter les objections possibles, qui empêchent de suivre la logique jusqu'au bout.

\section{Conditions nécessaires}

68 Sur le plan théorique, dès lors qu'on admet la facilité du langage technique, ces conclusions pédagogiques semblent s'ensuivre de manière presque inévitable. Il existe, pourtant, plusieurs objections importantes. Tout d'abord, concernant les capacités des étudiants, il faut reconnaître que l'exploitation des textes techniques serait difficile avec des étudiants qui n'auraient pas un niveau minimum en langue. Cette condition pose de sérieux problèmes, surtout en début de cursus. Pour éviter ce genre de difficultés, on pourrait envisager des exercices de rédaction sur des textes très courts, comme les " abstracts", les résumés, ou les introductions d'articles, en s'inspirant notamment des travaux de Swales (1990) ou de Morton (1999) sur la notion de « genre ».

D'autre part, l'idée de base étant de profiter des connaissances déjà acquises dans la discipline principale, il faut aussi supposer chez les étudiants des connaissances adéquates dans cette discipline. Si cela paraît raisonnable en deuxième cycle, ce n'est pas évident en DEUG, surtout pour les matières qui ne sont pas enseignées dans le secondaire. Personne ne conteste l'utilité, et même la nécessité, de connaissances disciplinaires chez l'enseignant de langue, même si, en règle générale, il n'est pas appelé à remplacer le professeur de la discipline principale. Selon Hutchinson \& Waters (1987: 163), il doit plutôt être en mesure de poser des questions intéressantes. Cependant, si son objectif est d'animer le cours en suscitant la discussion, les sujets proposés ne doivent pas dépasser les compétences des étudiants. Paradoxalement, ceci semble plus difficile avec des étudiants débutants qu'avec les plus avancés. Certes, il est possible dans un cursus 
bilingue d'aborder une nouvelle matière sans trop de difficultés, mais pour la grande majorité des étudiants, l'effet serait de mélanger les problèmes, de manière antipédagogique.

70 Afin d'éviter les problèmes liés à la connaissance de la discipline, on pourrait envisager l'utilisation de textes historiques, afin de profiter des connaissances générales. Malheureusement, les textes authentiques comportent souvent des archaïsmes linguistiques, qui risquent de compliquer inutilement la tâche. Pire, dans certaines disciplines, comme le droit, remonter dans le temps ne ferait qu'augmenter la complexité du contenu.

71 La deuxième catégorie d'objections concerne les spécificités de la discipline. Il faut admettre qu'en ce qui concerne la pédagogie linguistique, toutes les disciplines ne présentent pas les mêmes avantages. $\mathrm{Si}$, dans la plupart des disciplines scientifiques, les concepts techniques sont internationalement valables, il n'en va pas de même pour les disciplines juridiques; les concepts juridiques relèvent de traditions différentes, ce qui augmente les difficultés en ce qui concerne la compréhension.

Pour d'autres domaines professionnels, la langue spécialisée ne correspond pas à la définition du langage technique. Dans certains cas, on peut difficilement parler de langue de spécialité, puisque ni le vocabulaire ni les contraintes rhétoriques ne sont bien délimités. Ce qu'on présente par exemple comme la «langue des affaires » ne concerne pas uniquement la terminologie des contrats ou de la comptabilité; on remarque aussi dans de nombreux manuels des chapitres sur le « socialising ", le «small talk», et même le " eating out ». Dans ces conditions, il ne s'agit plus d'un sous-ensemble de la langue de référence ; sur le plan du vocabulaire et des tournures syntaxiques, cela relève plutôt de la langue de tous les jours ${ }^{8}$. De la même façon, ni la langue publicitaire ni la langue des relations publiques ne peuvent être considérées comme spécialisées; elles semblent plutôt constituer un reflet, plus ou moins fidèle, de la vie moderne.

Enfin, il existe aussi des objections externes, ou "extralinguistiques ", à l'utilisation systématique des textes techniques en cours de langue. En effet, la variété étant aussi un facteur important de motivation, il semble parfois préférable de proposer soit des textes de styles différents sur un même thème, soit un ensemble de textes du même type sur des sujets différents?

De la même façon, bien que l'on comprenne l'intérêt d'un cours fondé sur le langage technique, ou sur la terminologie de la discipline principale, une telle approche peut aussi sembler tristement utilitaire. Les étudiants savent, ou devraient savoir, que pour réussir dans la vie professionnelle, il ne suffit pas de bien connaître sa spécialité; il faut aussi avoir de la culture générale. Les étudiants, qui passent déjà de longues heures à étudier une discipline technique, réclament souvent la possibilité de faire autre chose pendant les cours de langue. Il n'est pas exclu de profiter du temps imparti pour traiter des sujets qui relèvent des institutions étrangères, ou pour aborder des questions de culture ou de société.

75 Ces objections montrent que les conclusions que l'on pourrait tirer sur le plan théorique ne sont valables que si certaines conditions nécessaires sont réunies. Elles montrent en même temps la spécificité, la difficulté, et l'intérêt du secteur LANSAD. En effet, une telle approche ne saurait convenir aux étudiants de LEA, car ces derniers, contrairement aux premiers, sont censés avoir déjà un niveau acceptable en langue/s, sans être spécialistes des matières « extralinguistiques ». 


\section{Conclusion} réponses définitives. Les objections envisagées n'obligent pas à abandonner la théorie ; elles obligent cependant à rechercher des compromis concernant les applications pédagogiques. Les conclusions devront donc être adaptées selon les situations particulières. empirique, de leur utilité pédagogique, il faudra trouver le moyen "d'objectiviser " l'évaluation des compétences. Le système d'évaluation CLES (Certification de la Compétence en Langue pour les Études Supérieures Spécialisées), dont le principe est adopté au niveau ministériel, sera ainsi appelé à jouer un rôle important pour la recherche en didactique linguistique.

La réforme pédagogique récente a rendu encore plus urgent le débat sur les spécificités du secteur LANSAD. En effet, la langue vivante étant désormais obligatoire dans toutes les différentes filières, il faudrait adapter l'enseignement en conséquence. Malheureusement, il n'y a toujours pas de consensus concernant les principes didactiques à adopter. En l'absence d'un programme susceptible de répondre aux besoins spécifiques dans le secteur, de nombreux étudiants risquent de garder leur niveau de faux-débutant. Au lieu de profiter des cours proposés, ils considéreront la langue comme un obstacle supplémentaire, ce qui ne pourra que compliquer la tâche du pédagogue.

Le modèle linguistique de la pragmatique intégré, même simplifié, a néanmoins permis de montrer l'impossibilité d'un travail efficace sur la langue sans la prise en compte du contexte et des intentions communicatives. De ce point de vue, le débat entre les tenants de l'enseignement terminologique et ceux qui préconisent plutôt l'enseignement de la langue générale semble avoir lieu sur de fausses bases. En effet, il faut éviter de voir le contenu disciplinaire et le rattrapage linguistique comme deux choses séparées. Selon les principes proposés, il ne peut être question de choisir entre deux méthodes considérées toutes les deux comme insatisfaisantes; il faut plutôt chercher à intégrer des approches didactiques interdépendantes.

80

Cette discussion aura, espérons-le, permis de faire avancer le débat en précisant les problèmes théoriques, et en montrant ainsi l'absence de certitudes en didactique. Elle a permis également de faire apparaître l'importance de la linguistique pragmatique pour la recherche sur la technicité et sur les langues spécialisées, comme pour la réflexion sur la didactique linguistique.

\section{BIBLIOGRAPHIE}

Birch, S. 1996. « French researchers publishing in English: an analysis of first drafts ». ASp 11-14, 75-88. 
Carston, R. 1998. « Negation, presupposition and the semantics/pragmatics distinction ». Journal of Linguistics 34, 309-50.

Charnock, H.R. 1982. « Technicité et facilité linguistique ». Les Langues modernes 76, 27-39.

Charnock, H.R. 1985. « La réflexivité en linguistique et dans le langage naturel ». Thèse, Université Paris 8.

Charnock, H.R. 1997. « Problèmes de métalangue - au delà de la grammaire ». LINX 36/1 Métadiscours et enseignement/apprentissage des langues, 55-69.

Charnock, H.R. 1998. «L'application de divers aspects de la linguistique à la didactique des langues : étapes et modalités ». In « La valorisation des compétences en langues » Actes du $25^{e}$ Congrès UPLEGESS, 79-91.

Cooke, R. 1993. « Learning to publish in English: how can French researchers bridge the gap?». ASp 1, 463-74.

Desmet, I. 1998. «Caractéristiques morphologiques, sémantiques, syntaxiques et discursives des vocabulaires spécialisés ", COFDELA Actes du $2^{e}$ colloque de Linguistique Appliquée, 292-305.

Ducrot, O et alii. 1980. Les mots du discours. Paris : Éditions de Minuit.

Dudley-Evans, T. \& M.J. St John. 1998. Developments in ESP: a multidisciplinary approach. Cambridge : Cambridge University Press.

Gauker, C. 1997. « Domain of discourse ». Mind 106, 1-32.

Guyon, E. \& A. Guyon. 1996. « Anglais de spécialité et plurilinguisme ». ASp 11-14, 1-12.

Halliday, M.A.K. \& R. Hasan. 1976. Cohesion in English. Londres : Longman.

Humbley, J. 1997. « Is terminology specialized lexicography?». Hermes 18.

Humbley, J. 1998. « Le terminologue et le spécialiste de domaine ». ASp 19-22, 137-49.

Hutchinson, T. \& A. Waters. 1987. ESP -a learning centred approach. Cambridge : Cambridge University Press.

Isani, S. 1993. « La langue de spécialité et les savoirs disciplinaires ». ASp 2, 199-208.

Lehmann, D. 1993. Objectifs spécifiques en langues étrangères. Paris : Hachette.

Lehmann, D. 1997. « English for specific purposes (ESP) et français sur objectifs spécifiques ». ASp 15-18, 11-26.

Lerat, P.1995. Les langues spécialisées. Paris : Presses universitaires de France.

Lerat, P. 1997. « Approches linguistiques des langues spécialisées ». ASp 15-18, 1-10.

Mathis, G. 1997. « Stylistique et discours scientifique ». ASp 15-18, 157-83.

Morton, R. 1999. « Abstracts as authentic materials ». ELT Journal 53/3, 177-82.

Mortureux, M-F. 1995. « Les vocabulaires scientifiques et techniques ». In J.Cl. Béacco \& S.

Moirand (dir.), Les enjeux des discours spécialisés. Cediscor/Paris 3, 13-26.

Récanati, F. 1989. « The pragmatics of what is said ». Mind and Language 4/4, 295-329.

Récanati, F. 1995. « The alleged priority of literal interpretation ». Cognitive Science 19, 207-32.

Récanati, F. 1996. « Domains of discourse ». Linguistics and Philosophy 19/5, 445-75, 1996.

Searle, J. R. 1979. « Le sens littéral ». Langue Française 42, 34-47. 
Swales, J. 1990. Genre analysis. Cambridge : Cambridge University Press.

Sweeney, S. 1997. English for Business Communication. Cambridge : Cambridge University Press.

Watson, J.D. \& F.H.C. Crick. 1953. « Molecular structure of nucleic acids: a structure for deoxyribose nucleic acid ». Nature 171, 737-38.

Widdowson, H.G. 1979. Explorations in Applied Linguistics. Oxford : Oxford University Press.

Widdowson, H.G. 1998. « Context, community and authentic language ». Tesol Quarterly 32/4, 705-16.

\section{ANNEXES}

Texte 1 : Le Nouvel Observateur 1997, No. 1723 : 26.

On peut aussi y écouter les CD audio, visionner des films au format DVD ou encore lire CDRom et DVD-Rom. Un système de téléphone mobile mains libres, via un GMS intégré et entièrement piloté du micro-ordinateur (à base de Pentium MMx cadencé à $200 \mathrm{MHz}$ ) complète l'équipement. Ah, dernier détail, ce terminal, équipé d'un fax-modem, permet la communication par courrier électrique ainsi que la connection à Internet... (cité par Mathis, G. 1997. « Stylistique et discours scientifique », ASp 15-18, 157-83).

Texte 2 : Hawking, S. \& Ellis.1973. The large-scale structure of space-time, Cambridge : CUP, 57.

A manifold corresponds naturally to our intuitive ideas of the continuity of space and time. So far this continuity has been established for distances down to about $10-15 \mathrm{~cm}$. By experiments on pion scattering (Foley et al. 1967). It may be difficult to extend this to much smaller lengths as to do so would require a particle of such high energy that several other particles might be created and confuse the experiment. Thus it may be that a manifold model for space-time is inappropriate for distances less that $10-15 \mathrm{~cm}$. and that we should use theories in which space-time has some other structure on this scale. However such breakdowns of the manifold picture would not be expected to affect General Relativity until the gravitational length scale became of that order. This would happen when the density became about $1058 \mathrm{gm} \mathrm{cm}-3$, which is a condition so extreme as to be completely beyond our present knowledge.

Texte 3 : Loi sur la répression de l'ivresse publique et sur les débits de boissons. Loi du 1er octobre 1917, modifiée par les lois des 20 décembre 1933 et 24 septembre 1941.

Article 10 : - Tous cafetiers, cabaretiers, tenanciers de café-concerts et autres débiteurs de boissons à consommer sur place, qui, en employant ou en recevant habituellement des femmes de débauche ou des individus de mœurs spéciales, pour se livrer à la prostitution dans leurs établissements ou dans les locaux y attenant, auront excité ou favorisé la débauche, seront condamnés à un emprisonnement de six jours à six mois et à une amende de cinquante francs à cinq cents francs ( $50 \mathrm{fr}$. à $500 \mathrm{fr}$.).

Les peines ci-dessus pourront être portées au double, si les femmes de débauche ou les individus de mœurs spéciales, visées au paragraphe précédent, appartiennent à la famille du délinquant.

Texte 4 : European court report. Financial Times. 1/6/1994.

Where intellectual property rights conflict with the interests of free competition, intellectual property rights should take precedence, according to a preliminary report of 
the European Court of Justice...

The central issue was whether, and if so under what circumstances, a refusal to grant a copyright licence could constitute an abuse of a dominant position.

It was agreed a refusal to grant licences in itself did not constitute an abuse. But there would be occasions where such a refusal had been exercised under such special circumstances that it created an unacceptable obstacle to undistorted competition and therefore the copyright owners' rights should be restricted.

Texte 5 : Financial Times, 1996.

Public debate in the US is currently dominated by a single case : that of Ms Stella Liebeck, 81 , who was awarded $\$ 2.9 \mathrm{~m}$, of which $\$ 2.7 \mathrm{~m}$ was punitive damages- against McDonald's after she spilt a cup of coffee in her lap, causing third degree burns. She had lodged the cup between her thighs before driving away from one of the company's restaurants. The punitive award was later greatly reduced by a judge and the case subsequently settled out of court for an undisclosed sum of less than $\$ 600,000$.

That example has obscured the underlying trend on tort cases, those brought against doctors for malpractice, manufacturers for harmful products, and motorists for accidents.

The number of such cases has fallen since the late 1980s.

Texte 6 : A. Rusbridger, The Guardian, 1987.

Dogs throughout the country will be reacting with alarm at the little noticed judgement handed down in the Court of Appeal last Friday by a bench under Lord Lane, the Lord Chief Justice. The case concerned one Colin Higson, aged 31, of Bolton, who had been gaoled for two years for having sexual relationships with a Pyrenean Mountain dog. This was brave of him, but his wife, unimpressed, turned him over to the police.

The trial judge said that the sentence must reflect the court's disgust and disapproval. $\mathrm{Mr}$ Justic Leggat, disagreeing, said the only object of a sentence was to prevent the recurrence of an offence. When all was said and done, said his Lordship, it was the appellant and, indeed, his wife and not the dog who needed help.

Texte 7 : J.M. Keynes. 1936. Preface to the French edition of The General Theory of Employment, Interest and Money, p. xxxii-xxxiii

I have called my theory a general theory. I mean by this that I am chiefly concerned with the behaviour of the economic system as a whole, with aggregate incomes, aggregate output, aggregate employment, aggregate investment, aggregate saving rather than with the incomes, profits, output, employment, investment and saving of particular industries, firms or individuals, and I argue that important mistakes have been made through extending to the system as a whole conclusions which have been correctly arrived at in respect of a part of it taken in isolation. (repris dans Deconinck-Brossard \& Offerle. 1996. Reading economics, Paris : Ellipses, 34.)

Texte 8 : Merton, R.C. 1998. « Applications of option pricing theory: 25 years later ", American Economic Review 88/3, 323-49 (p. 329). (Nobel Prize lecture 9/12/97)

With the derivative security characteristics fully specified, we turn now the fundamental production technology for hedging the risk of issuing a derivative security and for evaluating the cost of its production. To locate the derivation in a more substantive framework, I posit a hypothetical financial intermediary that creates the derivative securities in principal transactions for its customers by selling them contracts which are its obligations. 
Texte 9 : Britten, S. Financial Times, 23/4/98.

Although my sympathies are with the « doves » on the Bank of England Monetary Committee, their argument that it would be undesirable to raise interest rates now and reduce them soon afterwards is a bad one. One of the virtues of monetary policy is that it can be changed when circumstances change, without the need for political egg on anyone's face.

The case for relying on monetary policy is probably valid in relation to normal business cycles. But suppose a country is not just in a temporary recession but in a long- lasting slump in which output remains well below capacity levels due to a deficiency of spending? What happens then if interest rates have already gone as low as they can, but money supply growth remains depressed and spending is still weak?

This is what Keynes called a « liquidity trap » and is more popularly known today as « pushing against a string ».

Texte 10 : The Economist, December 1994.

A deeper understanding of what makes people tick could transform economics. Fear of getting hurt. Lack of self control. Regret. A desire to be fashionable. Religious belief. Overconfidence. Bad habits. All are part and parcel of everyday life. They are not, however, part and parcel of everyday economics. Most contemporary economic theories assume that humans are rational, and so tend to ignore apparently irrational behaviour. It is unsurprising, then, that of all the criticisms made of economics, one of the most widely believed is that the dismal science misunderstands why people do what they do. Economics, it is alleged, dehumanises homo sapiens, reducing him to homo economicus, a creature that is little more than a robot, brutishly programmed to make as much money as possible. (repris dans Deconinck-Brossard \& Offerle 1996 : 36.)

Texte 11 : Russell, B. 1905. « On denoting », Mind. Reprinted in Russell, 1956. Logic and knowledge, Londres : Allen Unwin, 51.

According to the view which I advocate, a denoting phrase is essentially part of a sentence, and does not, like most single words, have any significance on its own account. If I say, "Scott was a man", that is a statement of the form "x was a man", and it has Scott for its subject. But if I say, "the author of Waverley was a man", that is not a statement of the form "x was a man", and does not have "the author of Waverley" for its subject. Abbreviating the statement made at the beginning of this article, we may put, in place of "the author of Waverley was a man" the following: "One and only one entity wrote Waverley and that was a man". (This is not so strictly what is meant as what was said earlier, but it is easier to follow.) ...

Texte 12 : Pinter, H. 1975. No man's land, Londres : Methuen, 21.

Hirst: It's a long time since we had a free man in this house.

Spooner: We?

H: I.

$\mathrm{S}$ : Is there another?

$\mathrm{H}$ : Another what?

S: People. Person.

$\mathrm{H}$ : What other?

S: There are two mugs on that shelf?

$\mathrm{H}$ : The second is for you.

S: And the first? 
H: Would you like to use it? Would you like some refreshment?

S: That would be dangerous. I'll stick to your scotch if I may.

H: Help yourself.

S: Thank you.

\section{NOTES}

1. Il ne s'agit pas ici d'une présentation caricaturale. Selon le PV de la réunion du sous-groupe GERAS-Économie, du 25-6 mars 98, une majorité des enseignants en Facultés de Sciences Économiques estimaient que : " pour être crédible, l'enseignement de l'anglais économique doit s'aligner sur le programme d'analyse économique». Les enseignants en LEA et en IUP soulignaient pourtant l'importance de «la formation à la compréhension et à l'expression orales». En Droit, on assiste depuis longtemps au débat équivalent entre les enseignants $d^{\prime}$ «anglais juridique» et ceux qui proposent plutôt l'«anglais pour juristes». Les textes actuellement en vigueur ne permettent pas de résoudre la question. Ils préconisent une formation qui comporte « la pratique d'au moins une langue étrangère sous ses différents aspects (lecture, compréhension, expression écrite et orale ...) », mais ils précisent en même temps que « les thèmes propres aux disciplines enseignées [sont] largement pris en compte » (Arrêté relatif au Diplôme d'Etudes Universitaires, 1997, Titre III, article 6).

2. La terminologie utilisée ici est celle proposée par exemple dans Ducrot et al. (1980).

3. Les problèmes concernant l'interdépendance du sens et de la signification, et les conséquences pour la description de la métalangue naturelle, sont traités dans Charnock (1985). Les problèmes de la délimitation du domaine de discours et de la contribution de la pragmatique à la construction du sens enrichi (" enriched meaning») sont traités à plusieurs reprises par Récanati (1989). Voir aussi Gauker, (1997) et Carston, (1998). Signalons par ailleurs que dans un article récent, Widdowson (1998) revient sur la distinction entre form et meaning, fondamentale à une certaine époque pour la présentation de l'approche communicative. Il propose actuellement une distinction plus subtile entre la signification, au niveau de ce qu'il appelle le text, et le sens, associé au discourse.

4. On trouve cependant un exemple célèbre (car exceptionnel) à la fin du bref article de Watson \& Crick (1953 : 737) : "It has not escaped our notice [sic] that the specific pairing we have postulated immediately suggests a possible copying mechanism for the genetic material ».

5. Mathis (1997 : 178), relevant « pas moins de sept sigles, une dizaine de termes techniques qui ne sont passés dans la langue courante ... que pour les initiés ", déclare alors que ce texte " aurait tout aussi bien sa place dans une revue spécialisée ». Le mode d'expression est pourtant loin d'être technique.

6. Il n'en va pas de même pour Humbley (1998: 140), qui admet que : « les langues de spécialité représentent des entités très différentes, très diversifiées ». À partir de cette constatation terminologique, il tire des conclusions pédagogiques: «Ce n'est pas une découverte pour le GERAS de constater que les problèmes linguistiques [sic] des juristes, des économistes, des scientifiques, des techniciens sont très différents ... ». Dans le secteur LANSAD, cette conclusion didactique est loin d'être évidente.

7. De même, dans l'échelle des compétences linguistiques « Eurocentres », le candidat qui «peut exprimer différents points de vue » ou encore des, « opinions » est placé au niveau « Mastery » (le niveau le plus élevé), alors que celui qui « peut comprendre des informations données de façon claire mais sans simplification et trouver différents moyens de formuler ce qu'il a l'intention de dire » n'a que le niveau : « Competent communication ».

8. Dudley-Evans \& M.J. St John (1998: 31) s'étonnent que: "One major change has been the emergence of Business English as a major strand of ESP teaching. Early ESP work was dominated by English 
for Science and Technology... ». Les auteurs semblent oublier que, à la différence de l'anglais des affaires, la langue utilisée en EST correspond le plus souvent à la définition du langage technique. 9. On trouve pourtant les textes 7 et 10 dans le même chapitre d'un manuel récent, sur l'anglais de l'économie. Seul le premier, de Keynes, correspond à la définition du langage technique ; l'autre, tiré de The Economist, relève plutôt de l'anglais journalistique. On ne voit aucun rapport entre les deux textes ni au niveau du thème traité, ni au niveau stylistique. Les critères didactiques ayant motivé ces choix sont difficilement perceptibles.

\section{RÉSUMÉS}

Selon les définitions généralement proposées, les différentes langues de spécialité se distinguent de la langue de tous les jours surtout par la présence d'items lexicaux/syntaxiques spécifiques. En revanche, le langage technique peut se définir d'une manière plus générale, indépendamment du sujet traité, à l'aide d'une analyse pragmatique et stylistique, en fonction des critères de la cohésion, de la cohérence et de la connotation. Il ressort de cette analyse qu'il existe des degrés de technicité. Le même modèle linguistique permet d'élaborer une définition de la facilité linguistique, en rapport étroit avec la technicité. Selon cette analyse, le langage technique semble présenter moins de difficultés d'interprétation linguistique que la langue de tous les jours, ce qui permet de tirer des conclusions concernant la part de la discipline principale dans l'enseignement de la langue aux étudiants d'autres disciplines.

According to generally accepted definitions, specialised languages are distinguished from ordinary language principally by the presence of specific lexico/syntactic items. Technical language, however, may be defined in a more general way, independently of the subject matter, through a pragmatic and stylistic analysis using the criteria of cohesion, coherence and connotation. This analysis demonstrates the existence of degrees of technicality. The same linguistic model is used to give a definition of fluency, closely related to that of technicality. This analysis, according to which technical language appears to be linguistically easier to understand than ordinary language, leads to pedagogical conclusions concerning the place of the specialist subject in language teaching with students of other disciplines.

\section{INDEX}

Mots-clés : cohérence, cohésion, connotation, didactique, facilité linguistique, langue de spécialité, sens, signification, technicité

Keywords : didactics, fluency, intentional meaning, semantic meaning, specialised language, technicality

\section{AUTEUR}

\section{ROSS CHARNOCK}

Ross Charnock a préparé sa thèse à l'EHESS, sous la direction du professeur Oswald Ducrot. Il est responsable depuis 1990 du Service Langues Vivantes à la Faculté de Droit, de Sciences 
Économiques et de Gestion de l'université de Rouen. Il a également enseigné en LEA et à l'École Supérieure d'Électricité. En plus de ses travaux en linguistique et en linguistique appliquée, il a publié de nombreux articles sur la musique classique dans la revue internationale spécialisée The Strad. Il assure actuellement la traduction anglaise d'un ouvrage spécialisé sur l'histoire de l'archet en France. Charnock@auphine.fr 